\title{
Rotational Energy Harvesting using Bi-Stability and Frequency Up-Conversion for Low-Power Sensing Applications: Theoretical Modelling and Experimental Validation
}

\author{
Hailing Fu*, Eric M. Yeatman \\ Department of Electrical and Electronic Engineering, Imperial College London, London, SW7 2AZ, United Kingdom
}

\begin{abstract}
Kinetic energy harvesting has drawn great attention in the past decade, but low-frequency and broadband operation is still a big issue which impedes this technology to be widely deployed in low-power Internet of Things applications. In this paper, theoretical modelling and experimental validation of a rotational harvester with bi-stability and frequency up-conversion is presented for harnessing low-frequency kinetic energy with a wide bandwidth. Piezoelectric transduction was adopted to convert the rotational kinetic energy into electricity. Distributed-parameter modelling was employed for analyzing the electromechanical dynamics of the bistable piezoelectric beam. Bistable and frequency up-converting behaviours were considered in the theoretical model by introducing two external input magnetic forces. Different oscillating modes were analyzed, showing the variation of power generation capability under different modes, and the advantage of operating in the periodic double-well mode. From the potential well study, we got a conclusion that for the same input magnetic force, periodic double-well mode is capable of achieving a larger vibration amplitude compared to a havester without bi-stability. Asymmetric potential well shapes were investigated. This asymmetric shape provides a way to stabilize the initiation position of the beam for each plucking cycle, and eventually to stabilized the output. Key design factors to control the oscillating modes were studied, providing a guideline for future design. An experimental study was conducted to verify the theoretical results. A close match was achieved. This bistable harvester demonstrated a significant improvement(up to $2 \times$ ) compared to a harvester without bi-stability over a wide bandwidth (from 1 to $11 \mathrm{~Hz}$ ) at low frequencies, when operating in the periodic double-well mode. This paper presents a detailed theoretical model and in-depth analysis of a bistable frequency up-converting harvester, providing a fundamental understanding and guidance for low-frequency broadband energy harvester design.
\end{abstract}

Keywords: Rotational energy harvesting, magnetic plucking, bistability, frequency up-conversion, piezoelectric

\section{Introduction}

Since the invention of the voltaic pile in 1800, batteries have become the essential power sources for an increasing variety of domestic and industrial applications, ranging from mobile phones to electric cars. With the development of modern battery technologies, the energy density of batteries has reached the level of 1000 's of $\mathrm{mW} \cdot \mathrm{h} \cdot \mathrm{g}^{-1}$ with simple structures and reasonable costs $[1,2]$. However, battery technology has progressed more slowly than other fields of electronics [3]. In consequence, batteries are normally regarded as bulky, expensive and toxic components. Another drawback of batteries is the limited duration, for which replacement or recharging is compulsory if long-term operation is required. When a large number of devices are installed in remote or inaccessible locations (the Internet of Things [4], or implanted bio-sensors [5]), the process is inconvenient, costly or time-consuming.

Energy harvesting, a technology to convert ambient environmental passive energy, including solar, thermal gradient and kinetic energy, into electricity, has been regarded as a sustainable energy supply and a potential alternative

\footnotetext{
${ }^{*}$ I am corresponding author

Email addresses: h.fu14@imperial ac.uk (Hailing Fu), e.yeatman@imperial.ac.uk (Eric M. Yeatman)
} 
to conventional batteries [6]. Among these energy sources, kinetic energy harvesting has drawn great research interest. Devices have been developed to harness vibration, human motion, rotation, airflow and ocean waves based on different transduction mechanisms, including piezoelectric, electromagnetic, electrostatic and triboelectric conversion $[7,8,9,10]$.

Initially, kinetic energy harvesters, mostly vibration energy harvesters, were designed as linear devices operating at resonance to maximize the harvested energy. However, resonant energy harvesters exhibit a narrow bandwidth, and for micro-scale devices, the resonant frequencies are typically more than 100's of Hz [11, 12]. Therefore, these harvesters are undesirable for practical cases where motions normally have a wide frequency spectrum at low frequency. Frequency tuning is one solution, in which the resonant frequency is tuned to match the excitation frequency [13, 14], but such devices are either at macro-scale [15], or difficult to tune from a high resonant frequency (e.g. $>100 \mathrm{~Hz})$ for micro devices to a rather low frequency (e.g. $<10 \mathrm{~Hz}$ ) because of geometrical or dynamical constraints [16].

In order to broaden the operating bandwidth, nonlinear behaviour, which is often regarded as unwanted and troublesome in practical applications, has been intentionally introduced in energy harvesters and intensively studied in the last decade [17, 18]. Harvesters using monostable and bistable characteristics have been developed to enhance the energy harvesting performance over a wide bandwidth. Hardening and softening effects are two typical operating modes for monostable energy harvesters (nonlinear harvesters with one potential well) [19]. These modes can be achieved by applying tensile or compressive pre-loads in the axial direction of cantilever structures [20] to extend the operating frequency bandwidth close to the resonant frequency of its linear counterpart. For monostable harvesters, there are two steady state solutions, namely high-energy state and low-energy state (a well-known character of the forced-Duffing's equation) [21]. It is critical to design the harvester to operate in the high-energy state to improve the harvesting efficiency. Zhou et al designed a hardening monostable energy harvester using a piezoelectric cantilever with a tip magnet and external adjustable magnets to enhance the operating bandwidth [22]. Improved output power and bandwidth were realized in the frequency up-sweep compared to the down-sweep. However, solutions to keep the system operating in the high-energy state were not discussed. Mallick et al presented a solution to switch a monostable harvester from the low-energy state to the high-energy state using an electrical control mechanism [23]. Although the high-energy state operation is maintained, additional monitoring and controlling modules are required, and extra power will be dissipated by this control mechanism.

Bi-stability is another non-linear behaviour that has been investigated intensively. Double potential wells are normally introduced in these devices using pre-loaded forces [24], magnetic levitation [25] or even residual stress generated by the fabrication process [26]. When these devices oscillate between the wells (snap-through effect), improved vibration amplitude and velocity can be achieved. This type of harvester exhibits an enhanced power generation capability over a broader frequency bandwidth. However, the performance is severely affected by the operating modes $[27,28]$. These devices can operate in a single-well (intra-well) mode, chaotic double-well (interwell) mode or periodic double-well mode according to the input excitation energy [29]. The performance can be inferior to their linear counterpart when operating in the single-well or the chaotic double-well mode [30]. Efforts have been made to maintain the system to operate in the periodic double-well mode even when the input is random. Lan and Qin developed a bistable energy harvester with a low potential barrier by introducing an additional attracting magnet near the unstable position of a harvesting beam [31]. This attractive force allows the harvester to travel between two stable positions with low input energy requirement. Tri-stable [32] and quad-stable [33] energy harvesters were also investigated based on the bistable design to further expand the adaptability of broadband energy harvesters to random and broadband ambient kinetic energy.

Efforts have also been made to increase the operation bandwidth using the transition regime in bistable harvesters and variable or adaptive potential functions. Tang et al. developed a nonlinear vibration energy harvester using a repulsive magnetic force [34]. The transition region between a monostable hardening configuration and a bistable configuration was adopted to achieve the optimal performance in terms of bandwidth and output power. Using the same idea, Ibrahim et al. established a comprehensive mathematical model to study the system dynamics [35]. The transition regime between the monostable and bistable configuration was confirmed to be the optimal condition theoretically. In order to maintain bistable harvesters to operate in the double-well mode, adaptive or variable potential functions have also been employed in many studies. Hosseinloo and Turitsyn [36] proposed a non-resonant buylow-sell-high strategy that significantly improved the harvester's effectiveness at low frequencies. Variable potential functions were designed for different beam vibration conditions. Simulation results confirmed the improved performance when subjected to different excitation conditions compared to its conventional bistable and linear counterparts. 
Yang and Towfighian presented a novel adaptable potential design for a bistable energy harvester [37]. A movable tip magnet controlled by a spring in parallel to the piezoelectric beam was designed to passively adjust the position of the tip magnet in the beam length direction according to the magnetic force between the tip and fixed magnets. Using this adjustable potential design, a two-fold increase in the frequency bandwidth was obtained compared to a bistable system with a fixed tip magnet.

In terms of random and broadband motions, frequency up-conversion is another strategy to tackle these challenges. Direct impact[38] or magnetic plucking [39] is normally adopted to activate these harvesters (transducers) with low and random input excitation frequencies. After each excitation, the transducer oscillates in a damped free-vibration mode before the subsequent excitation occurs. This mechanism converts the low-frequency input excitation into highfrequency vibration at resonance, showing a broad bandwidth at a frequency range lower than the resonant frequency [40]. However, these harvesters normally face a severe power fluctuation at high frequencies [41, 42] due to the interference between the vibrating transducer and the forthcoming driving force. Xue and Roundy studied the electromechanical dynamics of frequency up-converting harvesters using magnetic plucking [43]. The power fluctuation issue was also reported in an experimental study when the driving frequency was above $5 \mathrm{~Hz}$. A detailed explanation of the formation of the power fluctuation and its relationship with the resonant frequency of the transducer were introduced and discussed in [44]. Increasing the system damping (electrical or mechanical) was recognized as a potential solution for reducing the power fluctuation. Fu and Yeatman introduced the bistable behaviour into a frequency upconverting rotational energy harvester to improve the damping coefficient and output power at high frequencies [45]. However, factors to maintain the system's operation in the high energy orbit and design considerations for this type of harvester have not been discussed and investigated in detail.

In this paper, a comprehensive theoretical study is presented to understand the electromechanical dynamics of a broadband rotational energy harvester using bi-stability and frequency up-conversion. Two external magnetic forces were introduced for bistability and for frequency up-conversion respectively. The dynamics of the harvester operating in different oscillating modes were investigated, illustrating the variation of the performance. This variation also shows the importance of designing the harvester to operate in the high energy orbit (periodic double-well mode). Key design factors to determine the operating mode are discussed. An experimental validation was conducted to verify the theoretical analysis. This paper provides a comprehensive study of a rotational energy harvester using bistability and frequency up-conversion.

\section{Harvester Design and Operating Principle}

The schematic of the bistable rotational energy harvester is illustrated in Fig. 1. Rotational motion can be acquired from external rotating sources such as micro-air turbines, vehicle wheels or rotating machinery. A cuboidal magnet is attached on the rotating host as the driving magnet. Due to the low rotational frequency and the limited sized of the driving magnet, the eccentricity caused by the driving magnet on the rotor is ignored. Or multiple driving magnets arranged in a symmetrical manner can be adopted to eliminate the eccentric effect in applications where rotating speed is high, such as in a micro turbine. A piezoelectric cantilever beam is installed above the rotating host. At the free end of the beam, a tip magnet is placed. The magnetic force between the driving magnet and the tip magnet plucks the beam periodically. In each plucking cycle, the beam is bent by the force first; then the beam is released when the driving magnet rotates away, and the beam oscillates at resonance in the damped free-vibration mode before the next plucking cycle starts. This phenomenon is called frequency up-conversion, which allows the harvester to operate effectively at rotational frequencies much lower than its resonant frequency.

In this design, in order to enhance the power generation capability over a wider bandwidth, another fixed magnet is mounted above the tip magnet, as shown in Fig. 1. This magnet and the tip magnet create a repulsive magnetic force which introduces two stable positions (equilibrium states) for the beam, as illustrated in Fig. 1. The beam stays in either Stable Position 1 or Stable Position 2, when the system is static. Therefore, in dynamic conditions, there are different oscillating modes, including single well vibration and double well vibration in a periodic or chaotic manner. The stored energy in the beam varies significantly for different oscillating modes. For the periodic double-well mode, vibration amplitude and velocity are the highest among these three modes. More power can be converted by this motion. Hence, the bistable mechanism allows the frequency up-converting harvester to produce more power from rotation at low frequency when the harvester operates in the periodic double-well mode. 


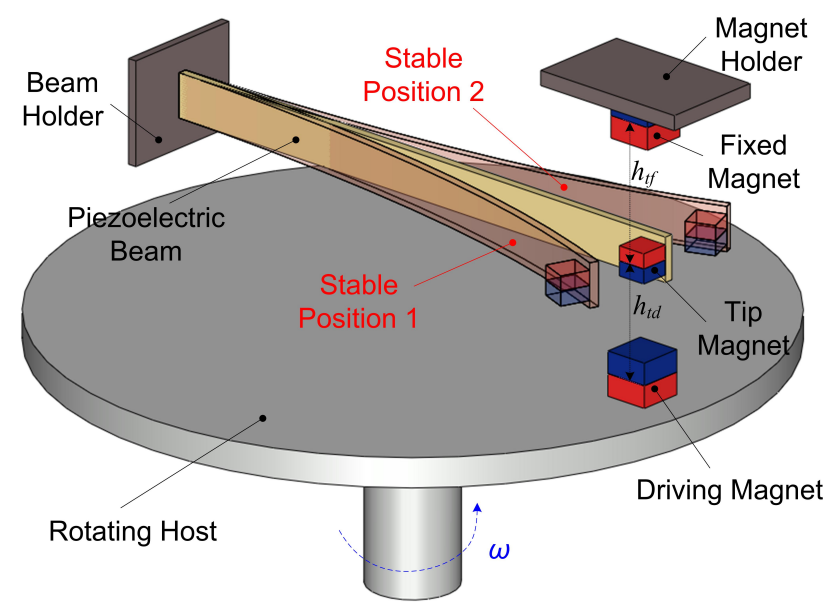

Figure 1: Schematic diagram of rotational energy harvester using frequency up-conversion and bistability. The transparent bent beams represent two stable positions of the beam. The red and blue colors in the magnets indicate the magnetization direction.

\section{Theoretical Modelling and Analysis}

The combination of bistability and frequency up-conversion makes the dynamics of the harvester more complicated. Therefore, it is necessary to build a theoretical model to study the electromechanical dynamics. Moreover, in order to enhance the performance of the harvester, periodic double-well vibration is required to generate a large vibration amplitude and velocity. In that sense, theoretical modelling is indispensable for the harvester design.

Fig. 2 depicts the force diagram of the system. Basically, there are two external magnetic forces $\left(F_{d t}\right.$ and $\left.F_{f t}\right)$ applied on the piezoelectric beam by the driving magnet and fixed magnet respectively. Fig. 2(b) shows the decomposition of external forces in each axis. In the z-axis, the forces are in opposite directions. Due to the similar magnet arrangements and dimensions, the resultant force in this direction is negligible. In the $\mathrm{x}$-axis, these magnets are well aligned initially when the beam is in the original shape $\left(d_{x 0}=0\right)$. Due to the small deformation of the cantilever beam, the limited gap generated by beam bending in the $\mathrm{x}$-axis is not enough to introduce a significant force component in this direction. Therefore, in this analysis, we mainly focus on the external forces $\left(F_{d t}^{y}\right.$ and $\left.F_{f t}^{y}\right)$ in the y-axis (beam bending direction) to study the electromechanical dynamics.

For the bistable beam in Fig. 1, the potential energy stored is the sum of the elastic potential energy $\left(U_{e}\right)$ of the beam and the magnetic potential energy $\left(U_{m}\right)$ of the tip magnet. Therefore,

$$
U_{t}=U_{m}+U_{e}
$$

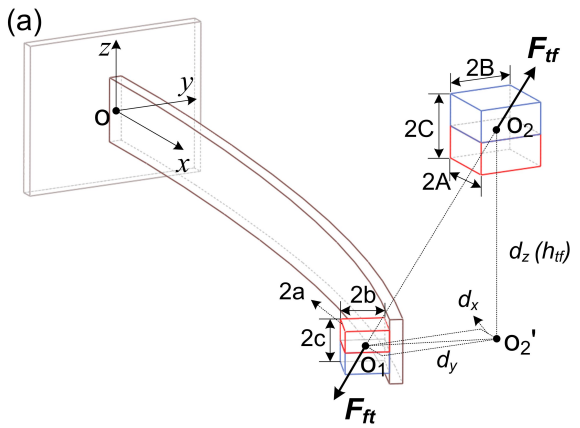

(b)

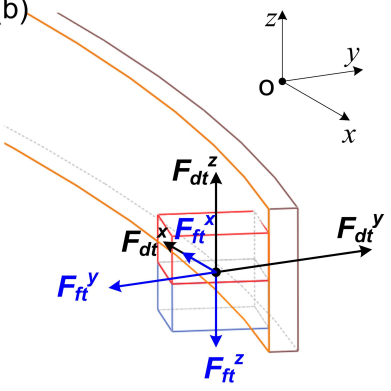

Figure 2: Force diagram of the bistable energy harvester. (a) Interaction between the tip magnet and fixed magnet. The force between them is repulsive. Dimensions and relative positions are illustrated for modelling. (b) Force diagram of the tip magnet enforced by the driving magnet and fixed magnet $\left(F_{d t}^{y}\right.$ means the force applied by the driving magnet on the tip magnet along the y-axis.). 
In order to calculate the magnetic potential energy stored in the magnets, a theoretical model proposed by Akoun and Yonnet is adopted [46]. The potential energy stored by the tip magnet can be expressed as

$$
U_{m}=\frac{J \cdot J^{\prime}}{4 \pi \mu_{0}} \sum_{i=0}^{1} \sum_{j=0}^{1} \sum_{k=0}^{1} \sum_{l=0}^{1} \sum_{p=0}^{1} \sum_{q=0}^{1}(-1)^{i+j+k+l+p+q} \cdot \psi\left(U_{i j}, V_{k l}, W_{p q}, r\right)
$$

where $J$ and $J^{\prime}$ are the magnetization of the magnets, $\mu_{0}$ is the magnetic constant, and $\psi$ is a function of the magnet dimensions and their relative positions in 3 axes. The function $\psi$ is given by

$$
\psi=\frac{U}{2}\left(V^{2}-W^{2}\right) \ln (r-u)+\frac{r}{6}\left(U^{2}+V^{2}-2 W^{2}\right)+\frac{V}{2}\left(U^{2}-W^{2}\right) \ln (r-V)+U V W \tan ^{-1}\left(\frac{U V}{r W}\right),
$$

where

$$
\begin{aligned}
& U_{i j}=d_{x}+(-1)^{j} A-(-1)^{i} a, \\
& V_{k l}=d_{y}+(-1)^{l} B-(-1)^{k} b, \\
& W_{p q}=d_{z}+(-1)^{q} C-(-1)^{p} c, \\
& r=\sqrt{U_{i j}^{2}+V_{k l}^{2}+W_{p q}^{2}},
\end{aligned}
$$

where $U_{i j}, V_{k l}$ and $W_{p q}$, correspond to the distances between the cube corners and their projections on the axes. The parameters $i, j, k, l, p$ and $q$, are equal to 0 or 1 according to the specific corner.

The magnetic force between two magnets in the y-axis can thus be calculated using

$$
F_{s t}^{y}=\frac{J \cdot J^{\prime}}{4 \pi \mu_{0}} \sum_{i=0}^{1} \sum_{j=0}^{1} \sum_{k=0}^{1} \sum_{l=0}^{1} \sum_{p=0}^{1} \sum_{q=0}^{1}(-1)^{i+j+k+l+p+q} \cdot \phi_{y}
$$

where $F_{s t}^{y}$ means the force applied on the tip magnet by one source (driving magnet or fixed magnet) in the y-axis, and

$$
\phi_{y}=\frac{1}{2}\left(U^{2}-W^{2}\right) \ln (r-V)+\frac{1}{2} r V+U W \tan ^{-1}\left(\frac{U V}{r W}\right)+U V \ln (r-U) .
$$

The elastic potential of the piezoelectric beam under bending motion can be given by

$$
U_{e}=\frac{1}{2} Y I \int_{0}^{L}\left[\frac{\partial^{2} v(x, t)}{\partial x^{2}}\right]^{2} d x
$$

where $Y I$ is the bending stiffness of the beam, and $v(x, t)$ is the beam transverse deformation.

In order to acquire the beam deformation, a theoretical model for analyzing its dynamics is necessary. Erturk and Inman developed an accurate model for piezoelectric beams under base vibration using a distributed-parameter method [47]. In our paper, adaptation has been made for the bistable frequency up-converting harvester model. The magnetic forces, $F_{d t}^{y}$ and $F_{f t}^{y}$, are considered as external forces applied at the free end of the piezoelectric beam, and the tip magnet is regarded as a proof mass.

Then, the dynamics of the bistable piezoelectric beam under tip excitation can be written as

$$
Y I \frac{\partial^{4} v(x, t)}{\partial x^{4}}+c_{s} I \frac{\partial^{5} v(x, t)}{\partial^{4} x \partial t}+c_{d} \frac{\partial v(x, t)}{\partial t}+m \frac{\partial^{2} v(x, t)}{\partial t^{2}}-\vartheta V(t)\left[\frac{d \delta(x)}{d x}-\frac{d \delta(x-L)}{d x}\right]=\left[F_{d t}^{y}(t)-F_{f t}^{y}(t)\right] \delta(x-L)
$$

and

$$
\frac{C_{p}}{2} \frac{d V(t)}{d t}+\frac{V(t)}{R_{l}}+\bar{e}_{31} h_{t} b \int_{0}^{L} \frac{\partial^{3} v(x, t)}{\partial x^{2} \partial t} d x=0
$$

where $c_{s} I$ is the internal damping, $c_{d}$ is the external air damping, $m$ is the mass per unit length of the beam, $\delta(x)$ is the Dirac delta function, $\vartheta$ is the piezoelectric coupling term in physical coordinates, $V(t)$ is the voltage across a resistive 
load $R_{l}, C_{p}$ is the inherent capacitance of the piezoelectric beam, $\bar{e}_{31}$ is the piezoelectric constant, $h_{t}=\left(h_{p}+h_{s}\right) / 2, b$ is the width of the beam and $L$ is the length of the beam. Details about the solution of the equations can be found in $[47,48]$. Using the above theoretical models, the electromechanical behaviour of the bistable beam can be acquired.

The average output power of the harvester on a resistive load $R_{l}$ is given by

$$
P_{\text {avg }}=\frac{\omega}{2 \pi} \int_{t_{0}}^{t_{0}+\frac{2 \pi}{\omega}} \frac{V^{2}(t)}{R_{l}} d t,
$$

where $\omega$ is the rotational frequency of the driving magnet. $R_{l}$ is the resistive load connected to the piezoelectric beam. Due to the nature of frequency up-conversion, the beam always oscillates at resonance even the driving frequency varies in a wide range. We assume the output impedance of the piezoelectric harvester is not affected by the driving frequency. A fixed load resistance value $\left(R_{l}=150 \mathrm{k} \Omega\right)$ is used in both the numerical study and experimental validation. This fixed value was chosen from an impedance matching measurement.

\section{Dynamics of bistable rotational energy harvester}

The theoretical equations in Section 3 are modeled in Matlab/Simulink, as shown in Fig. 3. This model includes mainly three modules, including the mechanical equation, electrical equation and the input force module. Three vibration modes are considered in this calculation. A set of parameters are given in Table 1 for numerically investigating the dynamics. The transducer is an customized piezoelectric beam from Johnson Matthey, and the material is M1100. The potential energy, operating modes, beam vibration displacement, output voltage and output power are studied.

\subsection{Potential energy of bistable harvester}

For potential energy analysis, only the elastic energy and the magnetic potential energy matter. Therefore, the driving magnet is not considered here. The potential energy is depicted in Fig. 4. As illustrated in this figure, there are two potential wells as a result of the combination of the elastic potential energy and the magnetic potential energy.

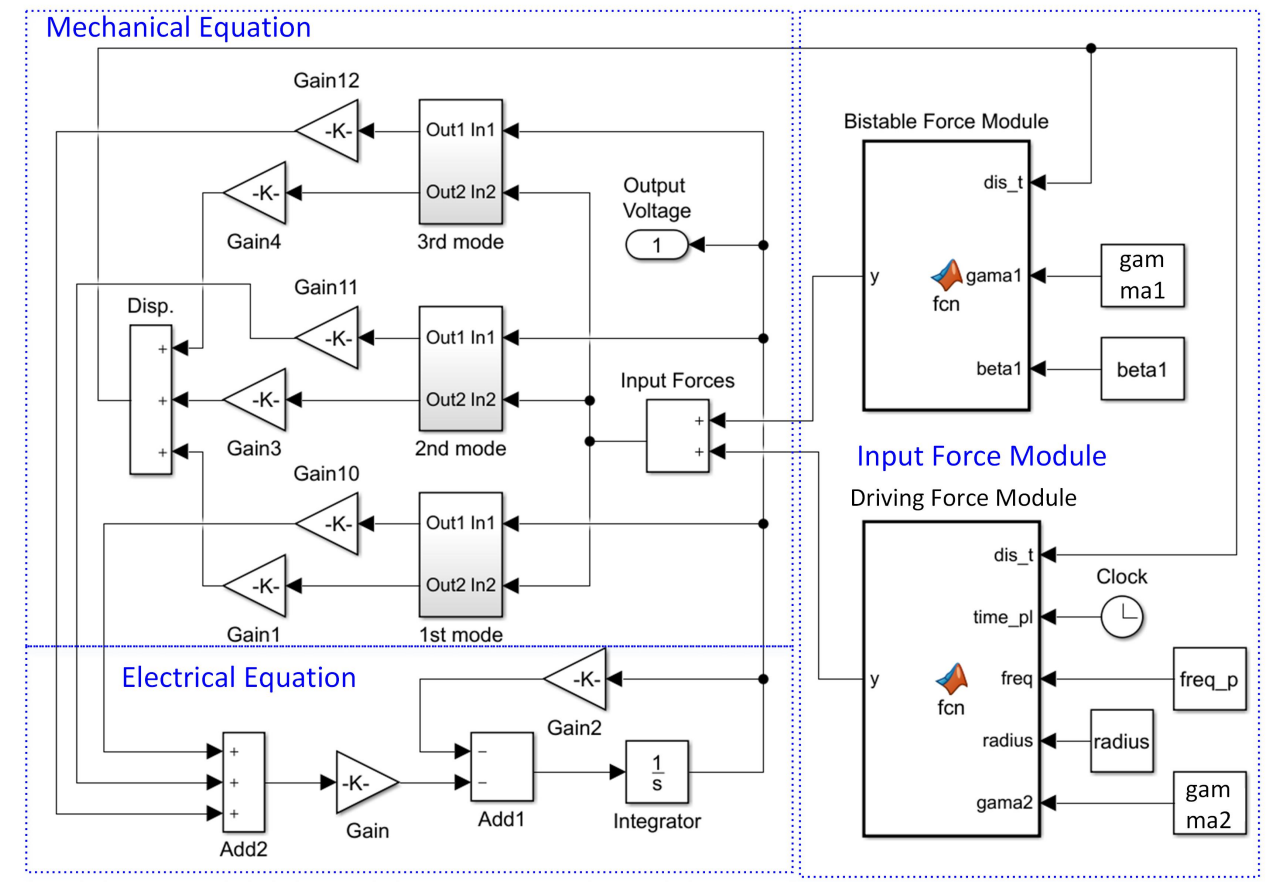

Figure 3: Simulink model for the frequency up-converting harvester. Three modules, including the mechanical and electrical equations and the input force module, are illustrated. Information, including input forces, beam displacement and output voltage can be acquired in the time domain. 
Table 1: Parameters for piezoelectric rotational bistable energy harvesters

\begin{tabular}{lll}
\hline \hline Symbol & Description & Value in Calculation \\
\hline$L$ & Length of beam & $33.5 \mathrm{~mm}$ \\
$b$ & Width of beam & $2 \mathrm{~mm}$ \\
$h_{p}$ & Thickness of piezoelectric layer & $0.15 \mathrm{~mm}$ \\
$h_{s}$ & Thickness of substrate layer & $0.1 \mathrm{~mm}$ \\
$a \times b \times c$ & Dimension of tip magnet & $0.5 \times 0.5 \times 0.5 \mathrm{~mm}$ \\
$A \times B \times C$ & Dimension of fixed \& driving magnet & $0.75 \times 0.75 \times 0.75 \mathrm{~mm}$ \\
$B_{r}$ & Remnant flux density of magnets & $1.17 \mathrm{~T}$ \\
$\rho_{p}$ & Density of piezoelectric material & $7700 \mathrm{~kg} / \mathrm{m}^{3}$ \\
$r_{m}$ & Rotating radius of driving magnet & $12 \mathrm{~mm}$ \\
$\rho_{s}$ & Density of substrate material & $1500 \mathrm{~kg} / \mathrm{m}^{3}$ \\
$e_{31}$ & Piezoelectric constant & $-22.2 \mathrm{~V} \cdot \mathrm{m}^{3} \mathrm{~N}$ \\
$d_{31}$ & Piezoelectric charge constant & $-315 \times 10^{-12} \mathrm{~m} / \mathrm{V}$ \\
$\epsilon_{r 33}^{T}$ & Piezoelectric relative dielectric constant & 4500 \\
$Y_{s}$ & Young's modulus of substrate & $140 \mathrm{GPa}$ \\
\hline \hline
\end{tabular}

The potential depth $\Delta P$ determines the input energy required to enable the beam to vibrate between the potential wells. In addition, from this figure, we can also find that for the bistable harvester, the required input energy for the beam to reach a specific deflection level is lower than that of the same harvester without bi-stability. For example, in order to get a beam displacement of $2.5 \mathrm{~mm}$, the energy required for a normal piezoelectric beam $\left(\Delta P_{1}\right)$ is much larger than that $\left(\Delta P_{2}\right)$ of a bistable beam as shown in Fig. 4. This also explains that for the same input driving force, bistable harvesters can achieve a larger beam displacement and harvest more power when vibrating between two wells.

For the two potential wells, the potential depth $(\Delta P)$ is normally the same (i.e. the wells are symmetrical), but asymmetrical shapes can be achieved by adjusting the magnetic potential. Fig. 5(a) - (c) shows the method to do this. An initial offset $\left(\Delta d_{t f}\right)$ in the beam bending direction is set between the fixed magnet and tip magnet. This initial offset changes the equilibrium positions of the beams, resulting in the variation of the potential well shapes. Fig. 5(d) depicts this variation for different values of offsets. The potential depths change for each well: one becomes deeper;

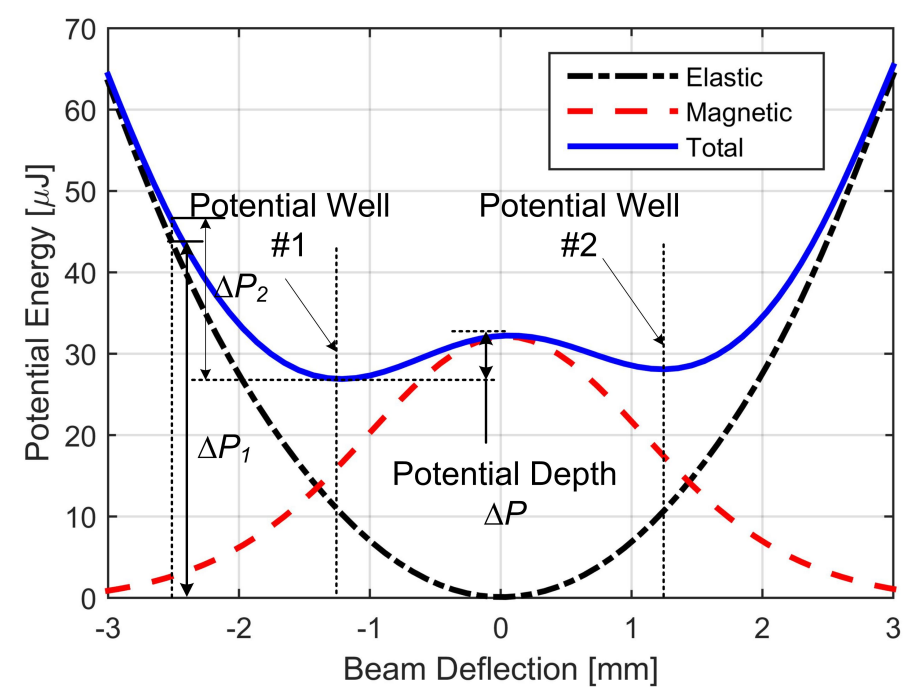

Figure 4: Potential energy of the bistable rotational energy harvester. The gap between the fixed magnet and the tip magnet $h_{f t}$ is $2.5 \mathrm{~mm}$. There is no gap initially between the fixed magnet and the tip magnet in the beam bending direction when the beam is not bent. 

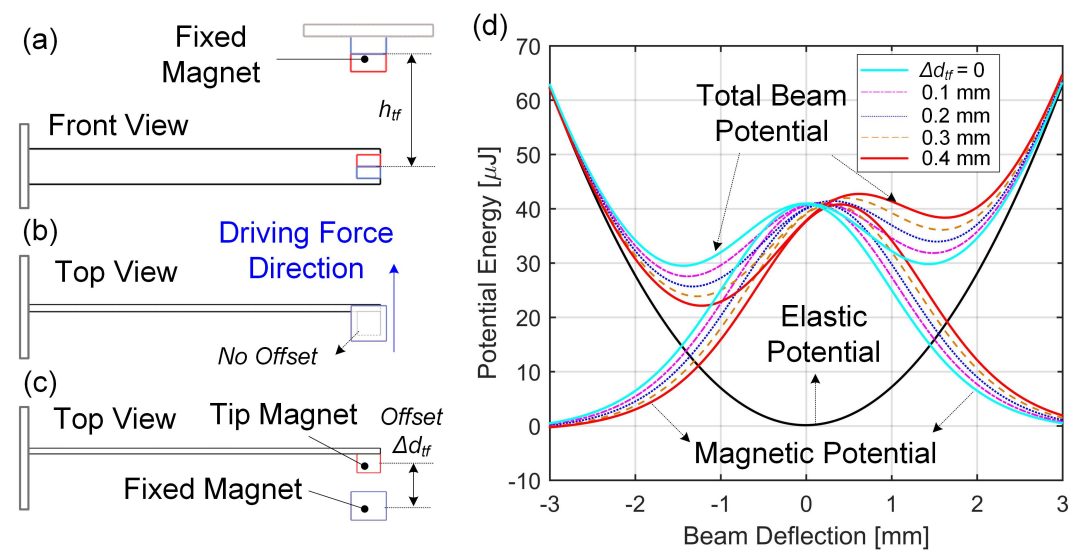

Figure 5: Introduction of asymmetrical potential wells for the harvester. (a) Front view of the beam, tip magnet and fixed magnet arrangement. (b) Top view of the arrangement (with no offset between the tip magnet and fixed magnet in the beam bending direction). (c) Top view with an offset $\left(\Delta d_{t f}\right)$. (d) Potential energy of the harvester with different offsets in the beam bending direction.

the other shallower. Overall, the required input energy increases in order to maintain the system operating between two wells, which is a disadvantage to the system, especially for inertial energy harvesting, where the driving force direction is alternating, and lower potential barriers are desirable.

However, for rotational energy harvesting, the driving force on the tip magnet is always in the same direction for each plucking when rotational motion is continuous. In this case, a fixed beam initiation position is beneficial to stabilize or even to improve the output performance. The asymmetric potential shape increases the probability of the system to settle into the potential well which has a higher potential barrier after each plucking. Therefore, a repeatable initiation or termination position can be obtained using the asymmetric potential well function.

\subsection{Operation mode of the harvester}

For this bistable frequency up-converting harvester, there are different operating modes. Considering the relationship between the input energy and the potential barrier, three oscillating modes, including single-well mode, vibrate in the double-well mode periodically, or vibrate chaotically between single-well or double-well modes, can be identified.

Fig. 6 shows these operation modes. When the input energy is not enough to conquer the potential barrier, the beam is confined in a single well. The beam displacement and velocity are limited. When the input is similar to the potential barrier, the system vibrates randomly between the single-well mode and double-well mode. The vibration displacement and velocity increase to some extent, but the performance is unstable and unrepeatable, as shown in Fig. 6(b) and the Poincaré map in Fig. 6(e). The system can also operate in the double-well mode periodically when the input energy is enough to penetrate the barrier for each excitation cycle. In this case, the performance is stable, and the duration for the beam to vibrate in the double-well mode is enhanced. The Poincaré map was plotted by indicating the beam's velocity and displacement at each of a set of specific time points in each excitation cycle. This map indicates the stability of the oscillation modes.

In addition, the mechanism of frequency up-conversion is also clearly illustrated in the displacement curves in Fig. 6(a), (b) and (c). The excitation frequency is $5 \mathrm{~Hz}$ for the driving magnet, but the beam is vibrating at resonance. For each excitation cycle, the beam is plucked by the magnetic force first, then oscillates at resonance before the second excitation approaches. For the case of the periodic double-well vibration, in each excitation cycle, the beam first oscillates between two wells for a few cycles. Then the beam settles into one well and operates in the single-well mode. The number of double-well vibration cycle for each plucking can be adjusted by changing the potential energy of the piezoelectric beam $\left(h_{t f}\right)$ or the input excitation $\left(h_{t d}\right)$ of the driving magnet.

For asymmetrical potential well arrangements, the dynamics of the system vary significantly. Fig. 7 depicts the dynamics of harvesters with different offsets between the tip magnet and fixed magnet in the beam bending direction. The initiation or termination position of each cycle for the asymmetrical configuration can be well controlled by setting appropriate offset values, whereas in the symmetrical case, the initiation or termination position alternates 

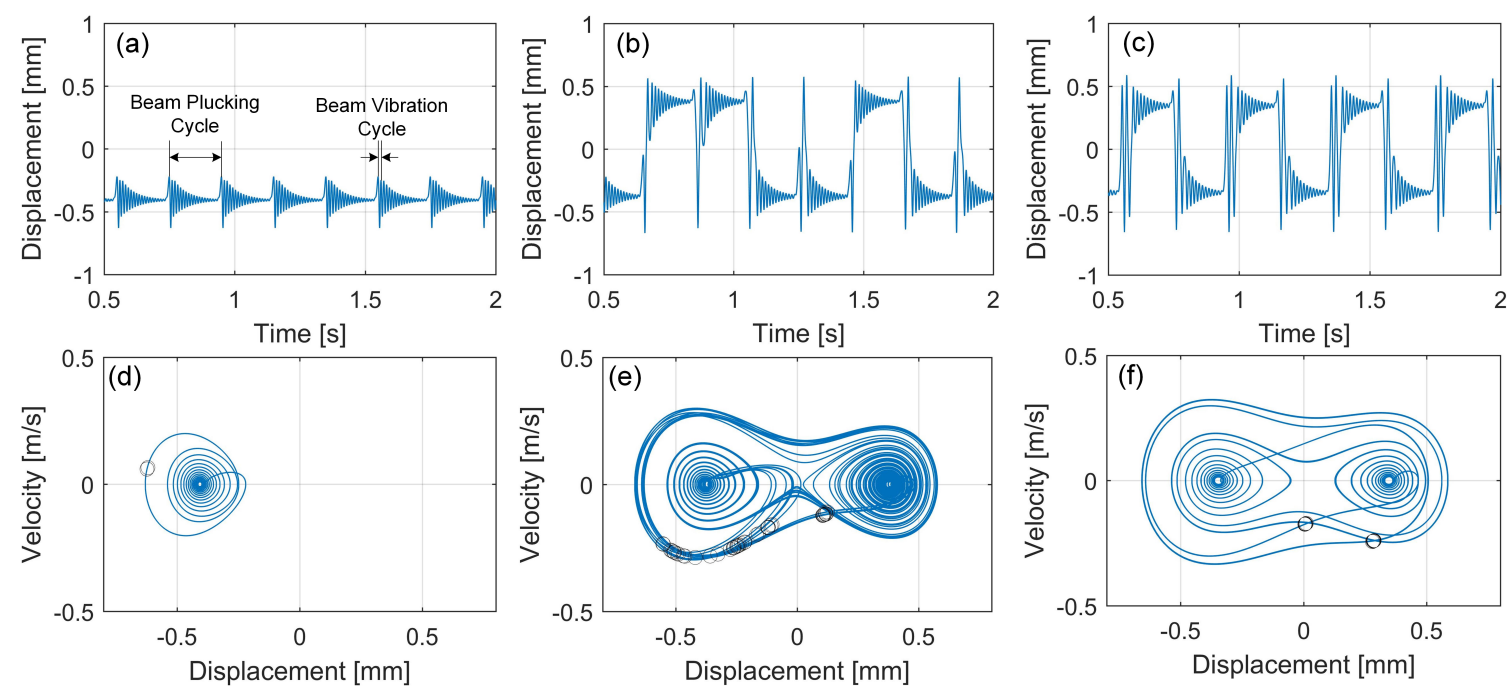

Figure 6: Beam tip displacement in time domain ((a),(b) and (c)) and velocity vs. displacement phase trajectory plots with an overlapped Poincaré map as black circles ((d), (e) and (f)) for three operating modes. (a) and (d) Single-well vibration. (b) and (e) Double-well chaotic vibration. (c) and (f) Double-well periodic vibration. In this calculation, the input force and driving excitation frequency for each mode were the same $\left(h_{t d}=3.2\right.$ $\mathrm{mm}$ and $\left.f_{e}=5 \mathrm{~Hz}\right)$. The magnetic potential energy $\left(h_{t f}=3.0,3.12\right.$ and $3.2 \mathrm{~mm}$ respectively.) was adjusted to get different modes.

randomly. This initial position also affects the input energy, and then the output power (as indicated in Fig. 7(c) and (f)). Although in both cases, the beam operates in the periodic double-well mode, the asymmetrical configuration exhibits a fixed initiation or termination position for the piezoelectric beam. This is ideal for the continuous rotation case to stabilize or even to enhance the output power (peak voltage amplitude: $3.9 \mathrm{~V}$ for Fig. 7(c) and $5 \mathrm{~V}$ for Fig. 7(f)).

With the variation of the offset value, the initial position of the beam changes from alternating between two wells to fixed at one well, as shown in Fig. 7(b) and (e). It is evident that during this transition, more complicated dynamic behaviours exist. It is worth studying the output of the harvester for different offsets $\left(\Delta d_{t f}\right)$ and also different gaps $\left(h_{t f}\right)$ between the fixed magnet and tip magnet to understand the dynamics better.
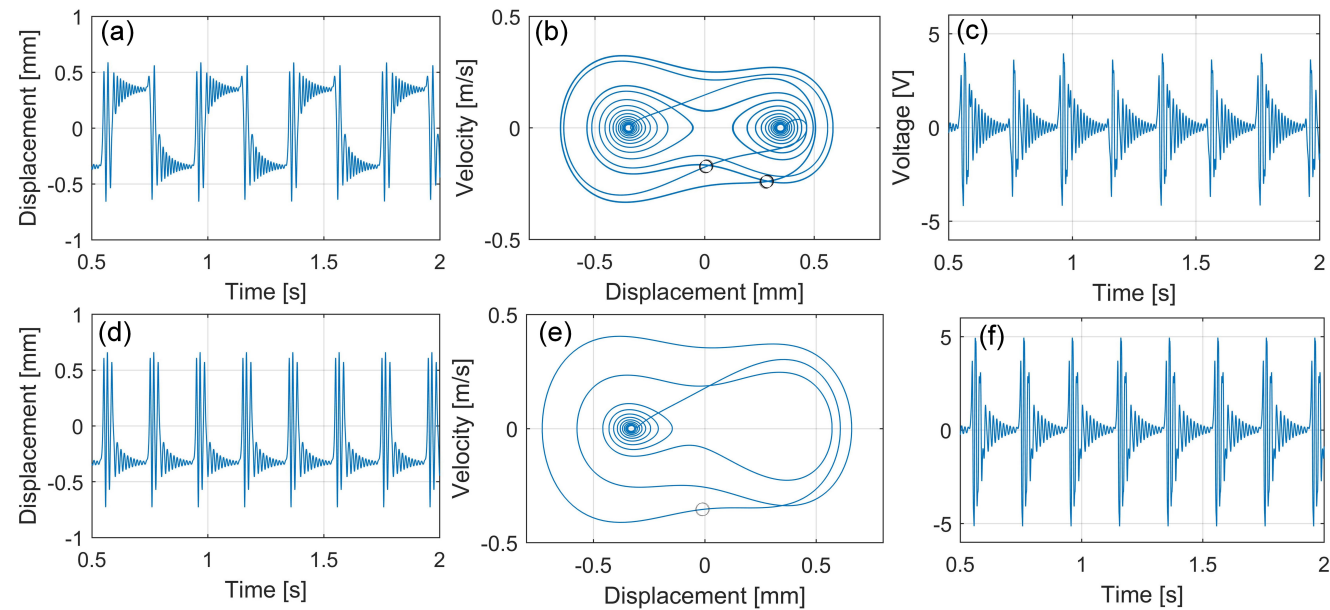

Figure 7: Beam displacement, velocity-displacement phase trajectory and output voltage for bistable energy harvesters with symmetrical or asymmetrical potential wells. (a), (b) and (c) are for $\Delta d_{t f}=0 \mathrm{~mm}$ : Symmetrical. (d), (e), and (f) are for $\Delta d_{t f}=0.1 \mathrm{~mm}$ : Asymmetrical. 


\subsection{Power output analysis}

The output power with different gaps in the z-axis and offsets in the y-axis is calculated using Eq. (10). Fig. 8 illustrates the variation of the output power for a given input force $\left(h_{t d}=3.2 \mathrm{~mm}\right)$ and different fixed-tip magnet arrangements. The transition of the oscillation modes from single-well vibration to periodic double-well vibration can be identified. From this figure, the best operation region for this given input can be also determined $\left(3.2 \mathrm{~mm}<h_{t f}<\right.$ $4 \mathrm{~mm}$ and $\left.-0.3 \mathrm{~mm}<\Delta d_{t f}<0.3 \mathrm{~mm}\right)$. The maximum output $(52.3 \mu W)$ happens at $h_{t f}=2.6 \mathrm{~mm}$ and $\Delta d_{t f}=0.2 \mathrm{~mm}$, but the performance in that local region varies significantly. This figure also indicates the significant impact of $h_{t f}$ and $\Delta d_{t f}$ on the performance. Appropriate parameters should be designed in order to ensure the enhanced output power.

It is evident that the input force also affects the dynamics of the harvester. By changing the relative gap $\left(h_{t d}\right.$ in Fig. 1), the input force can be adjusted. The variation of the output power as a function of the driving force (determined by $h_{t d}$ ) and force for bistability (determined by $h_{t f}$ ) is illustrated in Fig. 9. The variation of beam oscillation modes is indicated from the changes of the output power for different combinations of force from the driving magnet and force from the fixed magnet. The output is limited when the gap $h_{t f}$ is below $2.5 \mathrm{~mm}$. The beam operates in the single-well mode in this range. Then, the output experiences a chaotic increase when the gap $h_{t f}$ is in the range of $2.5 \mathrm{~mm}$ to 3 $\mathrm{mm}$, and the beam vibrates between single-well and double-well mode chaotically. When the gap $h_{t f}$ is larger than $3 \mathrm{~mm}$, the performance is more stable and enhanced. In this range, the double-well vibration is in operation. It is worth mentioning that the operating frequency in this calculation is fixed $(8 \mathrm{~Hz})$. This frequency affects the input energy of the piezoelectric beam. Therefore, it also affects the threshold for the beam to operate in the double-well mode. Therefore, an appropriate margin for the gap $h_{t f}$ compared to the threshold for the double-well operation should be considered, when the harvester operates with varying excitation frequencies.

Certainly, this transition is also relevant to the input driving force which provides the energy to conquer the potential barrier generated by the fixed magnet. As shown in Fig. 9, larger driving force (by reducing the gap $h_{t d}$ between the driving and fixed magnet) is desirable to achieve higher output power, but the elastic limit of the material should be considered to ensure the reliability of the system. If the stress generated on the beam by the driving force is

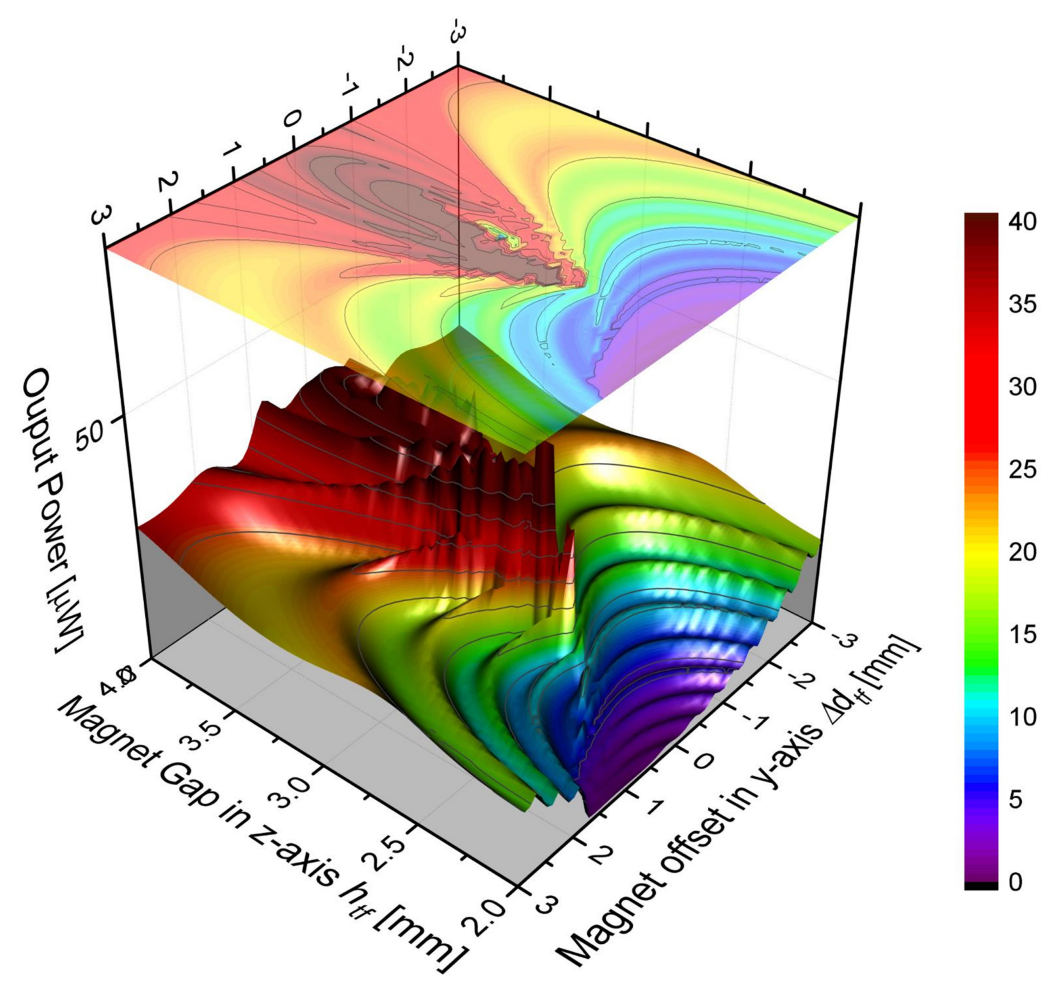

Figure 8: Output power for different gaps in the z-axis and different offsets in the $y$-axis between the tip and fixed magnet. The driving force and excitation frequency for this calculation are the same $\left(h_{t f}=3.2 \mathrm{~mm}, f_{e}=8 \mathrm{~Hz}\right.$ and $h_{t d}=3.2 \mathrm{~mm}$.). 


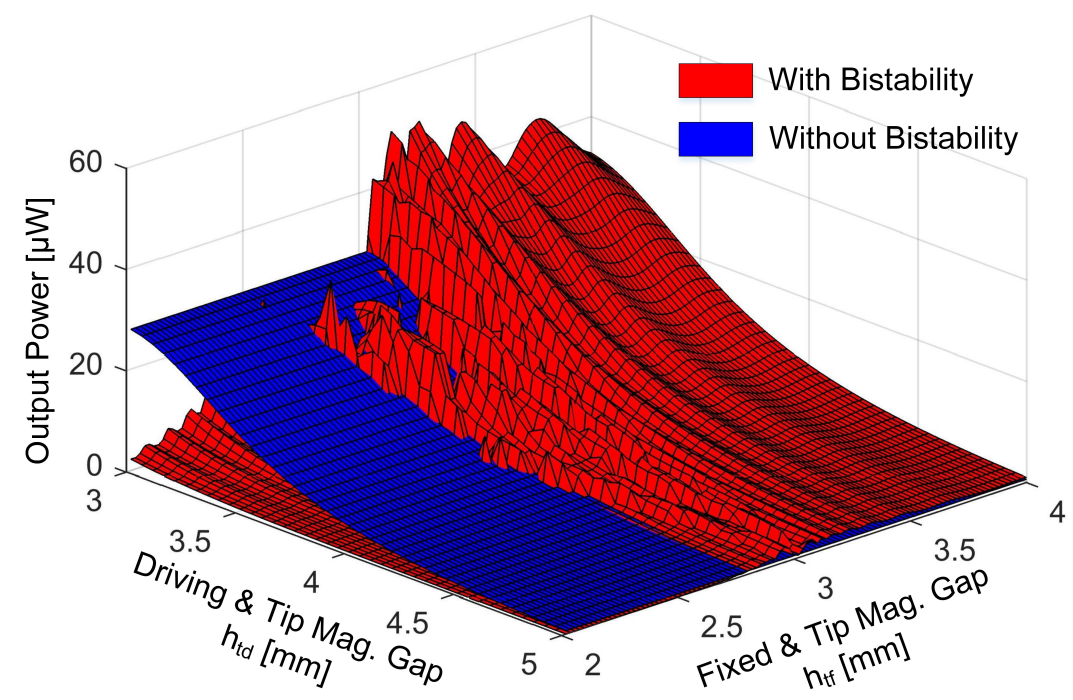

Figure 9: Output power for different driving forces (determined by the gap $h_{t d}$ between the tip and driving magnet) and forces for bi-stability (determined by the gap $h_{t f}$ between the tip and fixed magnet). The surface in red shows the output of the harvester with bi-stability, and the surface in blue is the output of harvester without bi-stability. This blue surface is extended from a single curve without fixed magnet (without $h_{t f}$ ), providing a reference for comparison. The calculation is performed at $8 \mathrm{~Hz}$. The offset between the fixed and tip magnets is fixed $\left(\Delta d_{t f}=0.1 \mathrm{~mm}\right)$.

close to or overpasses the elastic limit, the piezoelectric material faces a high possibility of degradation [49]. This is the reason why $3 \mathrm{~mm}$ is chosen as the minimum gap $\left(h_{t d}^{\min }\right)$ between the driving magnet and the tip magnet.

In order to illustrate the advantage of bistable mechanism in this rotational energy harvester, a comparison of output power between harvesters with (red surface) and without (blue surface) bi-stability is provided in Fig 9 as well. A distinct enhancement can be observed in this figure when the bi-stable harvester operates in the high energy orbit (double-well) for the same input driving force. This enhancement can be explained by the potential barrier reduction by the bistable mechanism. As shown in Fig. 4, to achieve a beam displacement of $2.5 \mathrm{~mm}$, the potential barrier for a bi-stable beam is much lower than that of a beam without bi-stability.

Another consideration for energy harvester design is the operating bandwidth. As in many practical applications, the excitation (rotational) frequency in the ambient environment is not constant, such as the rotational frequency of moving vehicles. Therefore, it is desirable to maintain high output power over a broad bandwidth. Fig. 10 provides a comparison of the output power between a bistable harvester and a harvester without bi-stability (without the fixed magnet in Fig. 1) as a function of driving \& tip magnet gap $h_{t d}$ and rotating frequency of driving magnet $f_{e}$. The frequency responses of two harvesters are illustrated and compared by adjusting the rotating frequency of the driving magnet. Two criteria, including output power and power fluctuation influence, are considered to determine the operating bandwidth for the bistable harvester.

A significant enhancement of output power is illustrated in the bistable harvester when the driving frequency is lower than $10 \mathrm{~Hz}$, over the whole range of the gap between the driving and tip magnets. It is ideal to operate this harvester below $10 \mathrm{~Hz}$. When the frequency is higher than $10 \mathrm{~Hz}$, the bistable harvester is inferior due to the power fluctuation issue. Considering the output power for both harvesters is sensitive to the input driving frequency, and varies significantly after $10 \mathrm{~Hz}$, this frequency bandwidth $(>10 \mathrm{~Hz})$ is not ideal for both cases. The reason the bistable harvester has a significant power decrease at high frequency is that the phase mismatching between the driving magnetic force and the motion direction of the vibrating beam [44] becomes severe at high frequencies. This mismatching affects the instantaneous input power to the piezoelectric beam. At a certain point (e.g. $12 \mathrm{~Hz}$ ), the input power is insufficient to conquer the potential barriers of the bistable beam. The beam, as a result, operates in the single-well mode with low output power. This explains the significant deterioration of the output power above $10 \mathrm{~Hz}$. Therefore, for this type of harvester, it is desirable to operate in the low-frequency range (e.g. $f_{e}<f_{r} / 10$, where $f_{r}$ is the resonant frequency of the piezoelectric beam.), and the bistable mechanism is beneficial to enhance 


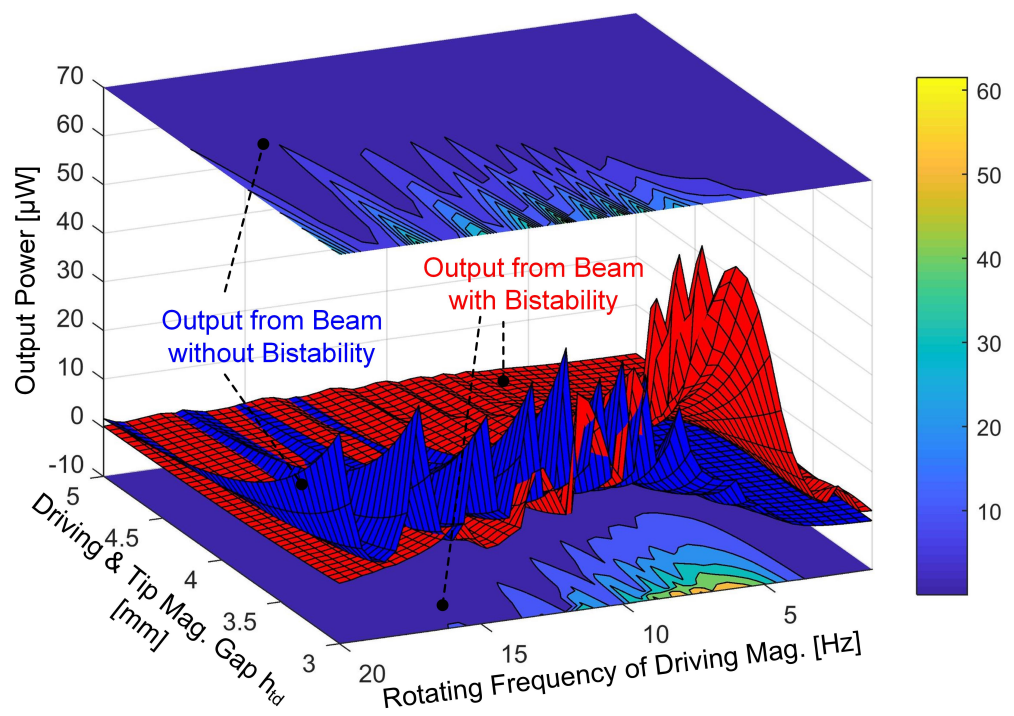

Figure 10: Output power versus the gap between driving and tip magnets and rotating frequency of the driving magnet. The surface in red shows the output of the harvester with bi-stability, and the surface in blue is the output without bi-stability. Contour plots for each surface are given. The gap and offset between the fixed and tip magnets are fixed $\left(h_{t f}=3.2 \mathrm{~mm}\right.$ and $\left.\Delta d_{t f}=0.1 \mathrm{~mm}\right)$.

the performance of the harvester at low frequencies.

For different beam dimensions, the upper limit of the operating frequency range can be determined by the transducer's natural frequency $f_{r}$ and the quality fact $Q$. For frequency up-converting harvesters, the tip displacement after each plucking is in the damped free vibration form (bi-stability is ignored in this analysis). The tip displacement $v(t)$ can be expressed as

$$
v(t)=\underbrace{v_{0} e^{-\pi \cdot f_{r} \cdot t / Q}}_{\text {Amplitude }} \cdot \underbrace{\cos \left(\omega_{d} \cdot t+\Phi_{0}\right)}_{\text {Frequency }},
$$

where $v_{0}$ is the initial beam vibration amplitude after each plucking, $f_{r}$ is the beam resonant frequency, $Q=1 /\left(2 \xi_{t}\right)$ is the transducer's quality factor, $\xi_{t}$ is total damping ratio, $\omega_{d}$ is the beam resonant frequency under damping and $\Phi_{0}$ is the initial phase of beam vibration. The vibration amplitude attenuation is determined by the "Amplitude" component in Eq. 11. If we consider the beam has to be damped to a percentage of $k$ to avoid the output power fluctuation, the relationship between the initial $v_{0}$ and final amplitude $v_{f}$ in one excitation cycle $\left(T=1 / f_{e}\right)$ can be written as

$$
k=\frac{v_{f}}{v_{0}}=e^{-\frac{\pi \cdot T \cdot f_{r}}{Q}}=e^{-\frac{\pi \cdot f_{r}}{Q \cdot f_{e}}}
$$

where $f_{e}$ is the excitation (plucking) frequency of the driving magnet. Then the upper limit $f_{e}^{u}$ of the driving frequency can be solved from Eq. 12 as

$$
f_{e}^{u}=-\frac{1}{\ln k} \frac{\pi}{Q} \cdot f_{r} .
$$

Therefore, the upper limit of the driving frequency $f_{e}^{u}$ is determined by both the system resonant frequency $f_{r}$ and also the quality factor $Q$. For a specific beam (with a fixed resonant frequency), a lower quality factor $Q$ (i.e. larger beam damping) is ideal to increase the frequency upper limit. For the beam damping, it includes mechanical damping and electrical damping. Hence, increasing the electrical damping will be the best option, as it not only increases the operating bandwidth, but also improves the output power (the extract power is normally regarded as the effect of the electrical damping on the beam). 


\section{Experimental validation}

\subsection{Experimental set-up}

An experimental set-up was built in order to implement the design and to verify the theoretical analysis, as shown in Fig. 11. In order to achieve a better control of rotational speed (driving frequency), a stepper motor (Phidgets 3303) with the step angle of $1.8^{\circ}$ was employed. The rotational speed can be adjusted accurately by a speed controlling circuit (Phidgets 1063) which has a position resolution of $1 / 16$ step. A driving magnet $(1.5 \times 1.5 \times 1.5 \mathrm{~mm})$ was mounted on a revolving host $(\varnothing 24 \mathrm{~mm})$ on the motor. A piezoelectric beam $(33.5 \times 2 \times 0.25 \mathrm{~mm})$ with a tip magnet $(1 \times 1 \times 1 \mathrm{~mm})$ was placed above the driving magnet, and fixed on a 3-axis linear positioning stage that can adjust the relative position of the beam. Another magnet $(1.5 \times 1.5 \times 1.5 \mathrm{~mm})$ for bi-stability was placed above the tip magnet on another positioning stage. Then the gaps among these magnets in each axis can be adjusted precisely. A laser detector was installed to measure the tip displacement of the piezoelectric beam. The beam was connected with a $150 \mathrm{k} \Omega$ resistive load. This resistance value was selected from an impedance matching test to maintain a reliable comparison between different configurations and operation conditions. The parameters of the piezoelectric beams are the same as those in Table. 1.

It is worth mentioning that the stepper motor, 3-axis linear positioning stages and the laser displacement sensor were adopted in this experimental set-up for the purpose of better and accurate control of the experimental study. For practical applications, the place of the rotating stage is taken by the rotating host structure, e.g. a turbine rotor, and the positioning stages and laser sensor are unnecessary. The bistable frequency up-converting harvester can be easily implemented within the dimensions of $\varnothing 35 \times 8 \mathrm{~mm}$ with the gaps between magnets considered. The overall dimensions can be further reduced by miniaturizing the piezoelectric transducer.

\subsection{Results and discussion}

Fig. 12 illustrates the dynamics of the harvester operating at different oscillation modes. Five oscillating modes were identified and studied in the experiment, including double-well vibration with varying initiation or termination position (positive or negative well, (a), (f) and (k)), double-well vibration with fixed initiation position either in the negative well ((b), (g) and (i)) or in the positive well ((c), (h) and (m)), and single-well vibration within the negative well ((d), (i) and (n)) or the positive well ((e), (j) and (o)). The dynamics vary significantly for different modes. When the harvester operates in the double-well mode, it has a high energy orbit ((f), (g) and (h)), which indicates that the output is higher. However, when the harvester operates in the single-well mode, the vibration displacement and output voltage are limited. Therefore, a reasonable relationship between the input force and the height of the potential barrier should be chosen to achieve effective harvesting. In addition, from the displacement-velocity portrait (f), (g) and (h), we can conclude that a stabler performance can be realized for the asymmetrical configurations (negative or positive well). A stable initiation or termination position (either negative or position potential well) has been realized in the asymmetric configurations, as shown in Fig. 12(g) and (h). The asymmetric configuration with a negative initiation

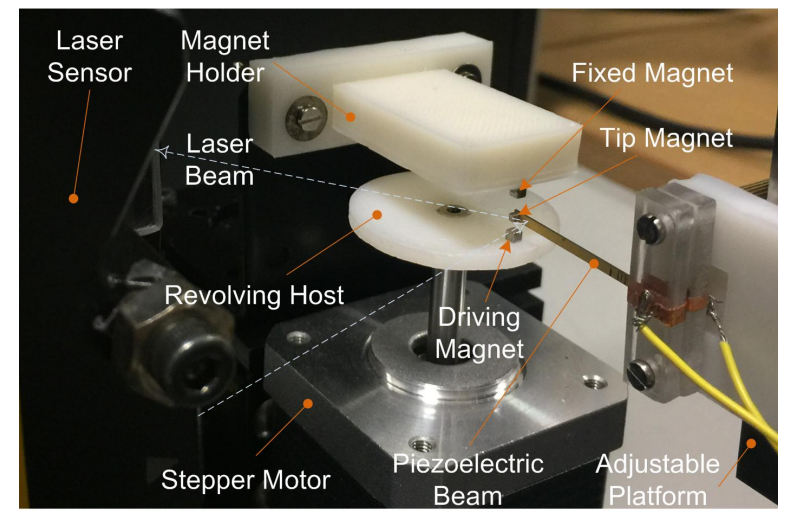

Figure 11: Experimental setup for the bistable frequency up-converting rotational energy harvester. 

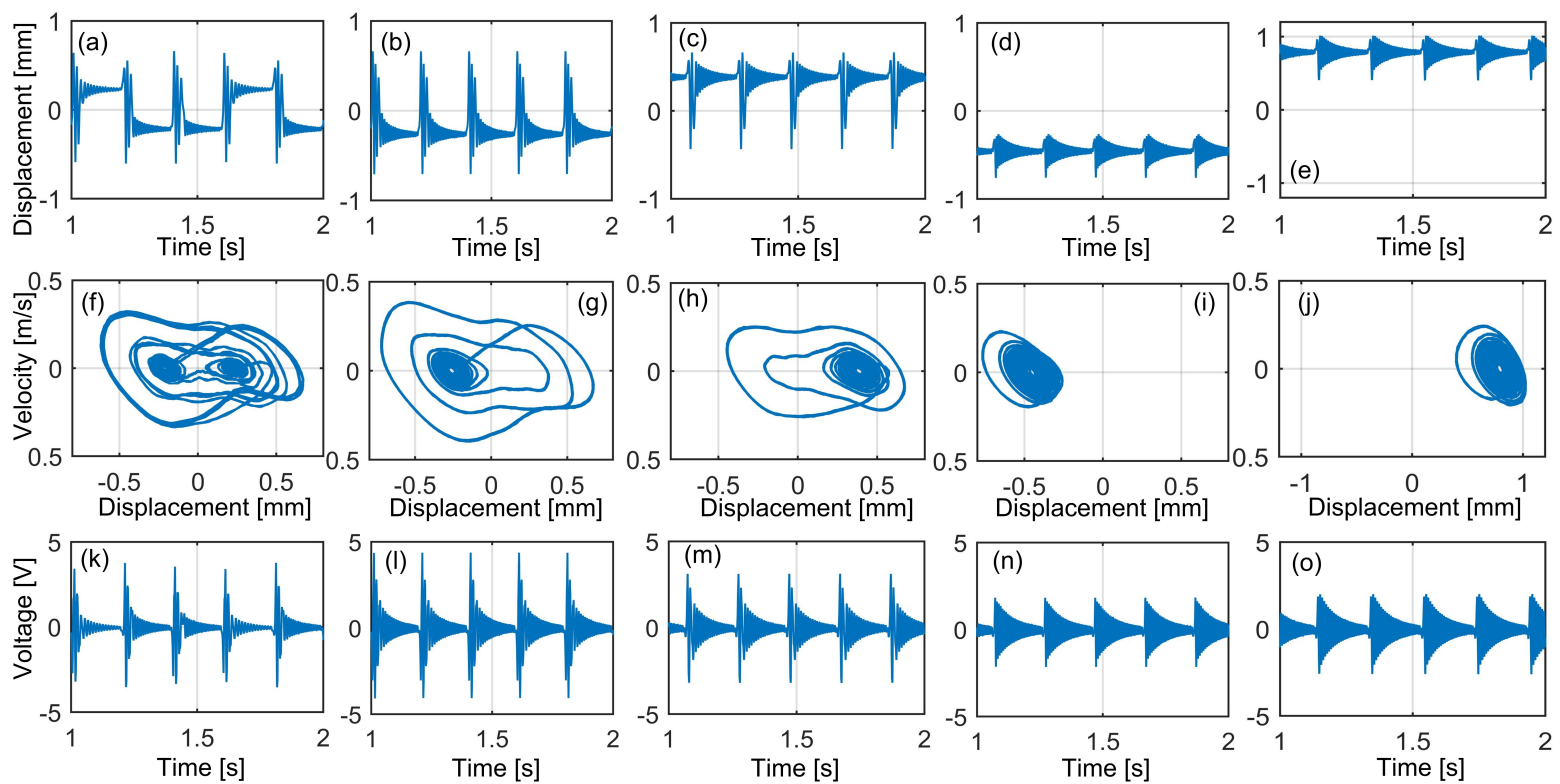

Figure 12: Experimental results: Dynamics of different operating modes, including beam tip displacement (a)-(e), displacement-velocity phase portrait (f)-(j) and output voltage on a $150 \mathrm{k} \Omega$ resistive load (k)-(o), with columns from left to right corresponding to $\Delta d_{t f}=0,0.1,-0.1,0.3$ and $0.4 \mathrm{~mm}$ respectively. The different operating modes were achieved by adjusting the relative offset $\Delta d_{t f}$ as shown in Fig. 5 . Other conditions stayed unchanged: $h_{t d}=4 \mathrm{~mm}, h_{t f}=3.2 \mathrm{~mm}$ and $f_{e}=5 \mathrm{~Hz}$.

position (the well away from the coming driving magnet) illustrates better energy harvesting performance (larger output voltage than others, as shown in Fig. 12(1)).

The experimental results fit well with the theoretical results in Fig. 6 and Fig. 7 in terms of vibration displacement and output voltage. The differences of the displacement-velocity phase portrait between theoretical and experimental results could be arisen from the piezoelectric hysteresis effect [50] which is ignored in the theoretical modelling and also from the inaccuracy of experimental parameters, but these factors do not have a severe impact on the outputs, and the validity of the theoretical model remains.

Fig. 13 provides a comparison of the tip displacement and output voltage between a bistable energy harvester operating in the periodic double-well mode with a fixed initiation position ((c), (d), (g) and (h)) and a harvester without bi-stability ((a), (b), (e) and (f)). The operating principle of the double-well mode is clearly demonstrated in Fig. 13(c) and (g). In one excitation cycle, the harvester first vibrates between two potential wells for a few cycles, and then settles in one stable position. In this case, the harvester always terminates in the negative potential well, and the next excitation cycle also initiates from this well. Therefore, this mode is called the double-well vibration with a fixed initiation or termination position. In addition, the concept of frequency up-conversion is also implemented as shown in these figures. The harvester is driven at $8 \mathrm{~Hz}$, but the cantilever beam always operates at its resonance. Theoretical ((a), (b), (c) and (d)) and experimental ((e), (f), (g) and (h)) results are compared for both beam oscillation conditions. These results have a close match in terms of beam vibration displacement, output voltage and vibration damping, further verifying the validity of the theoretical model.

Another significant phenomenon we can discover in this comparison is that the harvester with bistable mechanism can achieve a higher output voltage and damping coefficient by operating between two wells first and then settling in one fixed potential well. From the beam displacement and output voltage, the values in the beginning of each excitation are much larger for the bistable harvester than those for the harvester without bi-stability, resulting in an improved performance. At the end of each excitation, the displacement and output voltage from the bistable beam are much lower than those from the harvester without bi-stability. Therefore, the beam in the bi-stable harvester is more likely to be static before the forthcoming excitation force appears, avoiding the interference between the vibrating beam and the coming driving magnetic force, which might affect the performance. This interference can already be observed in the harvester without bi-stability in Fig. 13(a) and (e). If the driving frequency is higher, this interference 

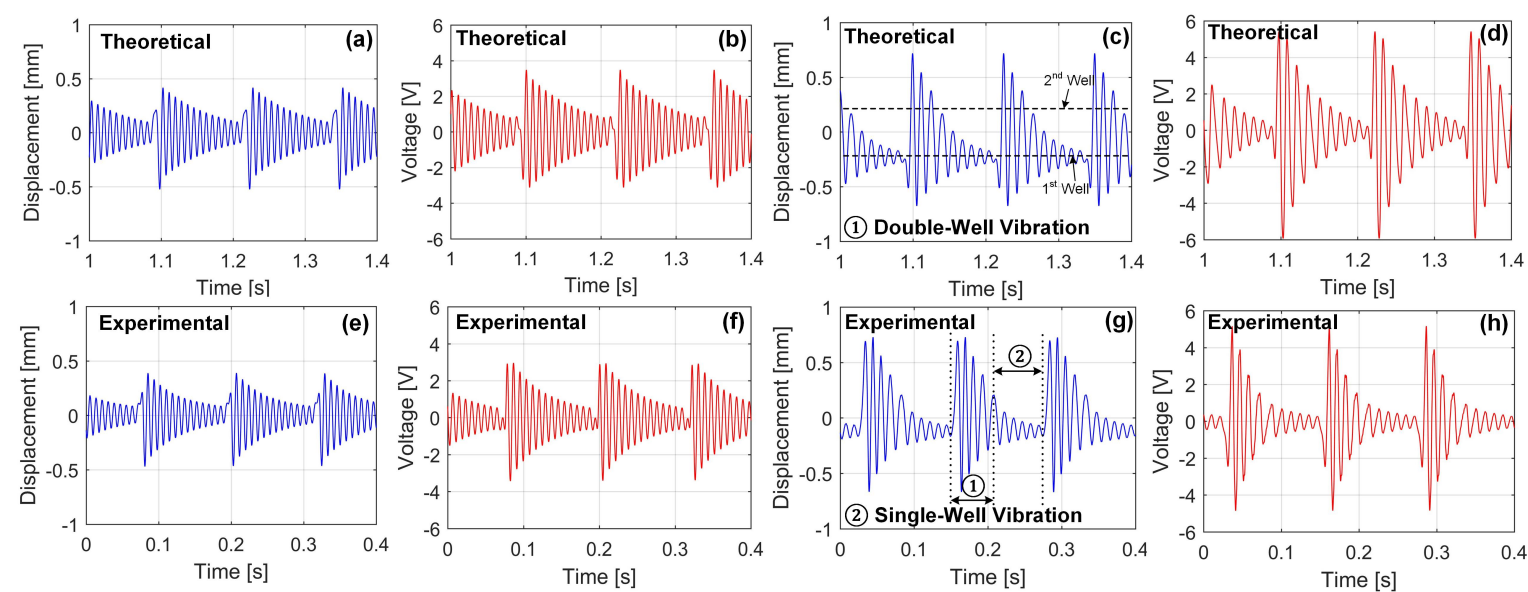

Figure 13: Comparison of the tip displacement and output voltage between a bistable beam operating at the double-well mode ((c), (d), (g) and (h)) and a harvester without bi-stability ((a), (b), (e) and (f)). Theoretical ((a), (b), (c) and (d)) and experimental (e), (f), (g) and (h) results are compared in both conditions. The driving frequency in this case was $8 \mathrm{~Hz}$, and the driving force remained the same $\left(h_{t d}=3.2 \mathrm{~mm}\right.$ and $\left.\Delta d_{t f}=0.1 \mathrm{~mm}\right)$.

will be more severe. In addition, the improved output voltage (Fig. 13(d) and (h)) is also beneficial to the rectification process if the power management circuit is considered. The improved voltage allows more charge to flow through the bridge rectifiers, so that more power can be stored by the power management circuit.

In order to examine the performance of the bistable harvester over a broad bandwidth, a frequency sweep test was conducted, as shown in Fig. 14. A constant rotational acceleration $\left(0.02 \mathrm{r} / \mathrm{s}^{2}\right)$ was adopted. This acceleration value can be controlled by the stepper motor controller (Phidget 1067). A low acceleration value was chosen to maintain the performance to be stable for all the frequencies.

As shown in Fig. 14, the output power fluctuates between 4 and $11 \mathrm{~Hz}$ for all configurations, including doublewell, signal-well and non-bistable harvesters. This fluctuation is caused by the interference between the vibrating beam and the subsequent driving force. As shown in Fig. 13(a) and (c), the beam vibrates freely at resonance after each plucking. The vibration amplitude attenuates gradually due to the system mechanical and electrical damping. When the driving frequency is high enough, the beam is still vibrating when the next driving force appears. The interference between the vibrating beam and the driving force due to phase mismatching causes the variation of the input energy to the piezoelectric beam, and results in the output power fluctuation. Solutions to this issue are:

(1) Use a lower driving frequency. This allows the beam to have a long period to be fully damped.

(2) Increase the electrical damping. This reduces the beam vibration at the point when the next plucking force appears, and reduces the interference. Also, the increased electrical damping also improves the energy harvesting performance. Using high-performance power management circuits is a way to improve the electrical damping.

The advantage of the bistable harvester operating in the double-well mode is evident over a broad bandwidth from 1 to $11 \mathrm{~Hz}$. This optimal operating bandwidth is determined by two criteria: (1) the output power should be enhanced in the considered frequency range compared to the harvester without bi-stability; and (2) the power fluctuation should not be severe enough to significantly affect the power output for different driving frequencies. In this range, the output power at some frequencies is two times higher than a harvester without bi-stability, such as at $10 \mathrm{~Hz}$. The general trend in this figure fits well with the theoretical analysis shown in Fig. 10. The differences in amplitude and frequency are caused by inaccurate structural and material parameters in the theoretical calculation compared to the practical harvester.

It is worth mentioning that the bistable harvester should be designed to operate in the double-well mode in order to improve the performance, because when it operates in the single-well mode, the performance is inferior to that of a harvester without bi-stability, as shown in the comparison in Fig. 14. For the single-well mode, the gap between the fixed and tip magnets is reduced $h_{t f}=2.7 \mathrm{~mm}$ compared to that in the double-well mode $\left(h_{t f}=3.2 \mathrm{~mm}\right)$. The potential barrier is much higher due to the reduced gap. The input energy, in this case, is not enough to conquer the barrier. The harvester, as a result, is confined in one of the two potential wells with reduced vibration amplitude and output power. 


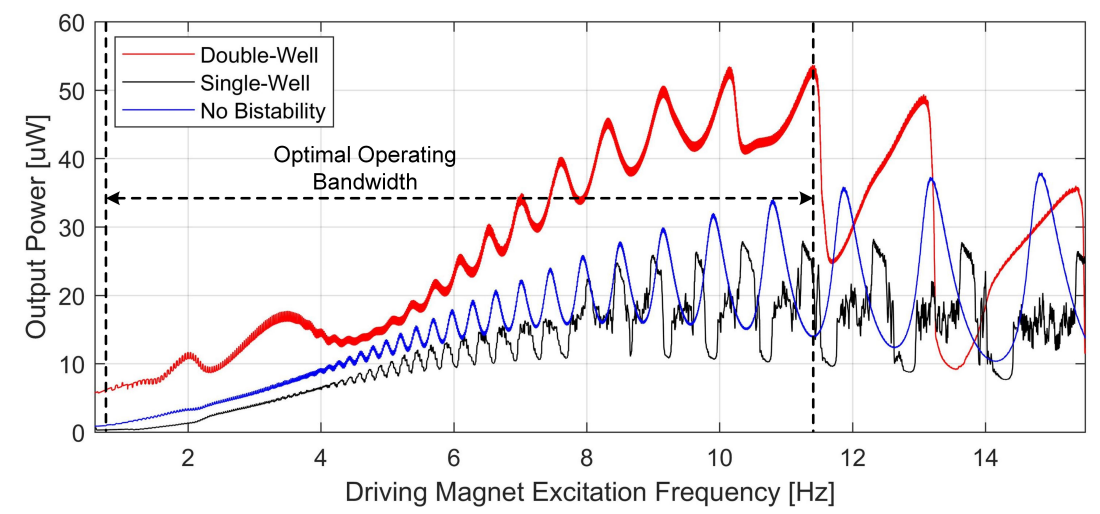

Figure 14: Output power versus driving frequency for bi-stable harvester operating at single-well and double-well mode and harvester without bi-stability. For all tests, the driving force was the same $\left(h_{t d}=3.2 \mathrm{~mm}\right)$. For the double-well mode, $h_{t f}=3.2 \mathrm{~mm}$ and $\Delta d_{t f}=0.1 \mathrm{~mm}$. For the single-well mode, $h_{t f}=2.7 \mathrm{~mm}$.

For the harvester with no bi-stability, the fixed magnet (as shown in Fig.1) is removed. Therefore, this harvester is a linear frequency up-converting harvester with only one potential well. Compared to the bistable harvester operating in the single-well mode, the shape of potential well is different, and the linear frequency up-converting harvester exhibits improved vibration amplitude and output power. Therefore, for bistable harvesters, the performance can be inferior when operating in the single-well mode. It is necessary to design the system properly to maintain the double-well operation.

When the frequency is higher than $11 \mathrm{~Hz}$, the performance of the bistable harvester operating in the double-well mode experiences significant variation and deterioration. The reason is that the interference between the vibrating beam and the subsequent magnetic force has a stronger effect on the instantaneous input power into the piezoelectric beam. This has been reflected by the power fluctuation between 4 and $11 \mathrm{~Hz}$ in Fig. 14. This interference becomes severe with the increase of the driving frequency. At a certain frequency, e.g. $12 \mathrm{~Hz}$, the input power is lower than the potential barriers of the bistable harvester. The harvester, as a consequence, operates in the single-well mode with limited output power. This explains the significant deterioration of the output power at high frequencies.

This power fluctuation issue also exists in harvesters operating in other modes or without bi-stability, which means for frequency up-converting harvesters, the driving frequency should be lower (e.g. $\left.f<f_{r} / 10\right)$ than the beam's resonant frequency. In fact, the operating bandwidth of this type of harvester is not limited in this range. We can extend the operating bandwidth to higher frequencies (e.g. $f_{\text {band }}=f_{r} / 8$ ) by increasing the electrical damping to attenuate the self-oscillation more quickly. Potential methods to increase the electrical damping includes more effective power management circuits, such as, voltage doubler circuits [51], pre-basing method [52] and synchronized switch harvesting on inductor method [53]. Further work will be conducted on this topic.

\section{Conclusions}

In this paper, a bistable frequency up-converting rotational energy harvester is studied theoretically and experimentally. Frequency up-conversion and bistable mechanisms were integrated to achieve effective energy harvesting at low frequency over a wide bandwidth. A theoretical model was established based on distributed-parameter modelling to study the electromechanical dynamics. Different operating modes were analyzed, showing the significant variation of the performance. Among them, the periodic double-well vibration shows a high energy orbit. In order to design the harvester to operate in this mode, different parameters including the relative position of the magnets, the driving force and driving frequency were investigated, providing a comprehensive understanding of the electromechanical behaviours. Asymmetric potential wells were achieved by adjusting the magnetic potential. This well shape provides a simple way to control the initiation (termination) position of the beam in each excitation cycle, enabling the harvester to have a stable and enhanced output and well-controlled behaviour.

An experimental validation was conducted to verify the theoretical analysis. A close match was achieved, showing the validity of the theoretical model. Different operating modes for the bistable harvester were also observed in the 
experimental study. A comparison between the bistable harvester operating in the double-well mode and a harvester without bi-stability was provided. The improvement of output voltage and damping coefficient of the bistable harvester operating in the double-well mode was explicitly illustrated. The harvester was finally tested in the frequency domain, and the dominant performance of the bistable harvester operating in the double-well at low frequency (1-11 Hz) mode was verified. Reasons for the power fluctuation and decrease was discovered and explained. Increasing the electrical damping is a potential solution to further increase the operating bandwidth. Future work will be focused on harvester output impedance study to obtain the maximum output power and effective power management circuit design to increase the electrical damping.

\section{Acknowledgement}

The work is supported by the China Scholarship Council (CSC) and the Department of Electrical and Electronic Engineering, Imperial College London. We would also like to thank Prof. Andrew Holmes for use of apparatus.

\section{References}

[1] D. Lin, Y. Liu, Z. Liang, H.-W. Lee, J. Sun, H. Wang, K. Yan, J. Xie, Y. Cui, Layered reduced graphene oxide with nanoscale interlayer gaps as a stable host for lithium metal anodes, Nature nanotechnology 11 (7) (2016) 626-632.

[2] T. M. Higgins, S.-H. Park, P. J. King, C. Zhang, N. McEvoy, N. C. Berner, D. Daly, A. Shmeliov, U. Khan, G. Duesberg, et al., A commercial conducting polymer as both binder and conductive additive for silicon nanoparticle-based lithium-ion battery negative electrodes, ACS nano 10 (3) (2016) 3702-3713.

[3] M. Armand, J.-M. Tarascon, Building better batteries, Nature 451 (7179) (2008) 652-657.

[4] J. Gubbi, R. Buyya, S. Marusic, M. Palaniswami, Internet of things (iot): A vision, architectural elements, and future directions, Future Generation Computer Systems 29 (7) (2013) 1645 - 1660.

[5] P. D. Mitcheson, Energy harvesting for human wearable and implantable bio-sensors, in: 2010 Annual International Conference of the IEEE Engineering in Medicine and Biology, 2010, pp. 3432-3436.

[6] S. Sudevalayam, P. Kulkarni, Energy harvesting sensor nodes: Survey and implications, IEEE Communications Surveys Tutorials 13 (3) (2011) 443-461.

[7] G. Gatti, M. Brennan, M. Tehrani, D. Thompson, Harvesting energy from the vibration of a passing train using a single-degree-of-freedom oscillator, Mechanical Systems and Signal Processing 66-67 (Supplement C) (2016) 785 - 792.

[8] C.-Y. Sue, N.-C. Tsai, Human powered mems-based energy harvest devices, Applied Energy 93 (2012) $390-403$.

[9] H. Fu, E. M. Yeatman, A miniaturized piezoelectric turbine with self-regulation for increased air speed range, Applied Physics Letters 107 (24) (2015) 243905.

[10] J. Chen, J. Yang, Z. Li, X. Fan, Y. Zi, Q. Jing, H. Guo, Z. Wen, K. C. Pradel, S. Niu, et al., Networks of triboelectric nanogenerators for harvesting water wave energy: a potential approach toward blue energy, ACS nano 9 (3) (2015) 3324-3331.

[11] E. E. Aktakka, K. Najafi, A micro inertial energy harvesting platform with self-supplied power management circuit for autonomous wireless sensor nodes, IEEE Journal of Solid-State Circuits 49 (9) (2014) 2017-2029.

[12] U. Bartsch, J. Gaspar, O. Paul, Low-frequency two-dimensional resonators for vibrational micro energy harvesting, Journal of Micromechanics and Microengineering 20 (3) (2010) 035016.

[13] C. Eichhorn, F. Goldschmidtboeing, P. Woias, Bidirectional frequency tuning of a piezoelectric energy converter based on a cantilever beam, Journal of Micromechanics and Microengineering 19 (9) (2009) 094006.

[14] G. Chen, Q. Meng, H. Fu, J. Bao, Development and experiments of a micro piezoelectric vibration energy storage device, Mechanical Systems and Signal Processing 40 (1) (2013) $377-384$.

[15] V. R. Challa, M. Prasad, Y. Shi, F. T. Fisher, A vibration energy harvesting device with bidirectional resonance frequency tunability, Smart Materials and Structures 17 (1) (2008) 015035.

[16] M. Lallart, S. R. Anton, D. J. Inman, Frequency self-tuning scheme for broadband vibration energy harvesting, Journal of Intelligent Material Systems and Structures 21 (9) (2010) 897-906.

[17] R. L. Harne, K. W. Wang, A review of the recent research on vibration energy harvesting via bistable systems, Smart Materials and Structures 22 (2) (2013) 023001

[18] T. Yildirim, M. H. Ghayesh, W. Li, G. Alici, A review on performance enhancement techniques for ambient vibration energy harvesters, Renewable and Sustainable Energy Reviews 71 (Supplement C) (2017) 435 - 449.

[19] L. G. W. Tvedt, D. S. Nguyen, E. Halvorsen, Nonlinear behavior of an electrostatic energy harvester under wide- and narrowband excitation, Journal of Microelectromechanical Systems 19 (2) (2010) 305-316.

[20] S. C. Stanton, C. C. McGehee, B. P. Mann, Reversible hysteresis for broadband magnetopiezoelastic energy harvesting, Applied Physics Letters 95 (17) (2009) 174103.

[21] P. L. Green, E. Papatheou, N. D. Sims, Energy harvesting from human motion and bridge vibrations: An evaluation of current nonlinear energy harvesting solutions, Journal of Intelligent Material Systems and Structures 24 (12) (2013) 1494-1505.

[22] S. Zhou, J. Cao, A. Erturk, J. Lin, Enhanced broadband piezoelectric energy harvesting using rotatable magnets, Applied Physics Letters 102 (17) (2013) 173901.

[23] D. Mallick, A. Amann, S. Roy, Surfing the high energy output branch of nonlinear energy harvesters, Phys. Rev. Lett. 117 (2016) 197701. 
[24] R. Masana, M. F. Daqaq, Electromechanical modeling and nonlinear analysis of axially loaded energy harvesters, Journal of Vibration and Acoustics 133 (1) (2011) 011007.

[25] M. Ferrari, V. Ferrari, M. Guizzetti, B. Ando, S. Baglio, C. Trigona, Improved energy harvesting from wideband vibrations by nonlinear piezoelectric converters, Sensors and Actuators A Physical 162 (2) (2009) 425-431.

[26] R. Xu, S.-G. Kim, Modeling and experimental validation of bi-stable beam based piezoelectric energy harvester, Energy Harvesting and Systems 3 (4) (2016) 313-321.

[27] R. Masana, M. F. Daqaq, Relative performance of a vibratory energy harvester in mono- and bi-stable potentials, Journal of Sound and Vibration 330 (24) (2011) 6036-6052.

[28] A. Erturk, D. J. Inman, Broadband piezoelectric power generation on high-energy orbits of the bistable duffing oscillator with electromechanical coupling, Journal of Sound and Vibration 330 (10) (2011) 2339-2353.

[29] R. Harne, K. Wang, On the fundamental and superharmonic effects in bistable energy harvesting, Journal of Intelligent Material Systems and Structures 25 (8) (2014) 937-950.

[30] B. Huynh, T. Tjahjowidodo, Experimental chaotic quantification in bistable vortex induced vibration systems, Mechanical Systems and Signal Processing 85 (Supplement C) (2017) 1005 - 1019.

[31] C. Lan, W. Qin, Enhancing ability of harvesting energy from random vibration by decreasing the potential barrier of bistable harvester, Mechanical Systems and Signal Processing 85 (Supplement C) (2017) $71-81$.

[32] S. Zhou, J. Cao, D. J. Inman, J. Lin, S. Liu, Z. Wang, Broadband tristable energy harvester: Modeling and experiment verification, Applied Energy 133 (Supplement C) (2014) 33 - 39 .

[33] Z. Zhou, W. Qin, P. Zhu, A broadband quad-stable energy harvester and its advantages over bi-stable harvester: Simulation and experiment verification, Mechanical Systems and Signal Processing 84 (Part A) (2017) 158 - 168.

[34] L. Tang, Y. Yang, C.-K. Soh, Improving functionality of vibration energy harvesters using magnets, Journal of Intelligent Material Systems and Structures 23 (13) (2012) 1433-1449.

[35] A. Ibrahim, S. Towfighian, M. I. Younis, Dynamics of transition regime in bistable vibration energy harvesters, Journal of Vibration and Acoustics 139 (5) (2017) 051008.

[36] A. H. Hosseinloo, K. Turitsyn, Non-resonant energy harvesting via an adaptive bistable potential, Smart Materials and Structures 25 (1) (2015) 015010.

[37] W. Yang, S. Towfighian, A hybrid nonlinear vibration energy harvester, Mechanical Systems and Signal Processing 90 (2017) $317-333$.

[38] L. Gu, C. Livermore, Impact-driven, frequency up-converting coupled vibration energy harvesting device for low frequency operation, Smart Materials and Structures 20 (4) (2011) 045004.

[39] A. M. Wickenheiser, E. Garcia, Broadband vibration-based energy harvesting improvement through frequency up-conversion by magnetic excitation, Smart Materials and Structures 19 (6) (2010) 065020.

[40] S. Chen, L. Ma, T. Chen, H. Liu, L. Sun, J. Wang, Modeling and verification of a piezoelectric frequency-up-conversion energy harvesting system, Microsystem Technologies 23 (7) (2017) 2459-2466.

[41] T. Galchev, H. Kim, K. Najafi, Non-resonant bi-stable frequency-increased power scavenger from low-frequency ambient vibration, in: TRANSDUCERS 2009 - 2009 International Solid-State Sensors, Actuators and Microsystems Conference, 2009 , pp. 632-635.

[42] Q. Tang, X. Li, Two-stage wideband energy harvester driven by multimode coupled vibration, IEEE/ASME Transactions on Mechatronics 20 (1) (2015) 115-121.

[43] T. Xue, S. Roundy, On magnetic plucking configurations for frequency up-converting mechanical energy harvesters, Sensors and Actuators A: Physical 253 (Supplement C) (2017) $101-111$.

[44] H. Fu, E. M. Yeatman, A methodology for low-speed broadband rotational energy harvesting using piezoelectric transduction and frequency up-conversion, Energy 125 (2017) 152-161.

[45] H. Fu, E. M. Yeatman, Broadband rotational energy harvesting with non-linear oscillator and piezoelectric transduction, Journal of Physics: Conference Series 773 (1) (2016) 012008.

[46] G. Akoun, J.-P. Yonnet, 3d analytical calculation of the forces exerted between two cuboidal magnets, IEEE Transactions on magnetics 20 (5) (1984) 1962-1964.

[47] A. Erturk, D. J. Inman, An experimentally validated bimorph cantilever model for piezoelectric energy harvesting from base excitations, Smart materials and structures 18 (2) (2009) 025009.

[48] A. Erturk, D. J. Inman, Piezoelectric energy harvesting, John Wiley \& Sons, 2011.

[49] P. Pillatsch, B. Xiao, N. Shashoua, H. Gramling, E. Yeatman, P. Wright, Degradation of bimorph piezoelectric bending beams in energy harvesting applications, Smart Materials and Structures 26 (3) (2017) 035046.

[50] Q. Xu, Identification and compensation of piezoelectric hysteresis without modeling hysteresis inverse, IEEE Transactions on Industrial Electronics 60 (9) (2013) 3927-3937.

[51] N. A. K. Z. Abidin, N. M. Nayan, M. M. Azizan, A. Ali, Analysis of voltage multiplier circuit simulation for rain energy harvesting using circular piezoelectric, Mechanical Systems and Signal Processing 101 (Supplement C) (2018) 211 - 218.

[52] J. Dicken, P. D. Mitcheson, I. Stoianov, E. M. Yeatman, Power-extraction circuits for piezoelectric energy harvesters in miniature and lowpower applications, IEEE Transactions on Power Electronics 27 (11) (2012) 4514-4529.

[53] A. Chérif, M. Meddad, S. Belkhiat, C. Richard, D. Guyomar, A. Eddiai, A. Hajjaji, Improvement of piezoelectric transformer performances using sshi and sshi-max methods, Optical and Quantum Electronics 46 (1) (2014) 117-131. 